\title{
Efficient Design of Fault Detection Architectures for Power Networks by using Game Theory *
}

\author{
D. Saracho ${ }^{* *}$ F. J. Muros ${ }^{*, * *}$ J. M. Maestre* \\ * Dep. of Systems and Automation Engineering, Univ. of Seville, Spain \\ ** e-distribution digital networks, Endesa, Enel group, Spain \\ (e-mail:dsarachov@gmail.com, \{franmuros,pepemaestre\}@us.es)
}

\begin{abstract}
In this paper, a ranking of the best locations to install remote control transformation centers (RCTCs) in distribution power lines is obtained by using the Shapley value of a cooperative game. The characteristic function of the game is based on both supply quality and economic indicators, which are relevant aspects regarding the operation and viability of electricity corporations. An academic example based on real data taken from a Spanish electricity company is considered to illustrate the feasibility of the proposed approach.
\end{abstract}

Copyright (C) 2020 The Authors. This is an open access article under the CC BY-NC-ND license (http://creativecommons.org/licenses/by-nc-nd/4.0)

Keywords: Distribution power networks, remote control transformation centers (RCTCs), cooperative game theory, Shapley value, supply quality indicators.

\section{INTRODUCTION}

Electricity is produced in power generation plants and then transported by transmission networks at high voltages to minimize energy losses. This power is then reduced to a lower voltage in electrical substations, so it can be used in homes and industry, being supplied to the final consumer by distribution power networks (Grainger et al., 2016; Gómez-Expósito et al., 2018). Due to its high impact on the cost of electricity and its direct correlation with customer satisfaction, the reliability of the distribution networks is a relevant topic in the electric power industry (Brown, 2009). While transmission networks are fully interconnected for the sake of reliability, providing redundant paths for the power to flow, distribution networks are operated in a radial fashion so that a fault only affects its own supply. In particular, when there is a fault in the electrical equipment, the recloser located at the beginning of the corresponding line isolates the electrical facility, allowing the rest of the distribution network to continue working normally as it is shown in Fig. 1.

In order to measure the power supply continuity and quality, it is possible to find several supply quality indicators in the literature, mainly based on the number/duration of the interruptions. Typical examples utilized worldwide are the System Average Interruption Duration Index (SAIDI) and the Average Service Avalaibility Index (ASAI) (IEEE, 2012). In this work, we will use the TIEPI (Spanish Ministry of Economy, 2000), which is the Spanish acronym for Interruption Time Equivalent to the Installed Capacity (ITEIC), a well-known legal indicator of quality of service used in Spain for distribution power lines. In particular, Spanish law defines ITEIC as a measurement of the time

\footnotetext{
* Financial support by the H2020 ADG-ERC project OCONTSOLAR (ID 789051), the MINECO-Spain project DPI2017-86918-R (C3PO), and the Andalusian Regional Government project US1265917 (GESVIP) is gratefully acknowledged.
}

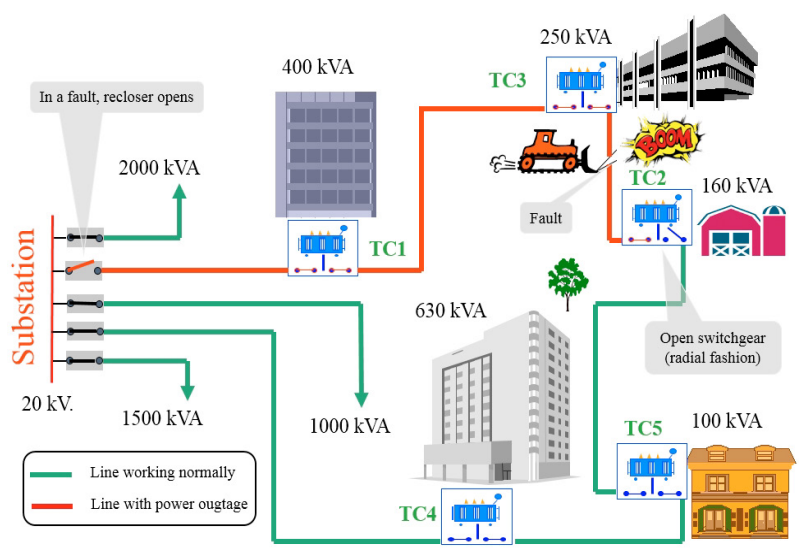

Fig. 1. Distribution networks in a radial fashion

of supply interruptions in terms of power interrupted. To this end, it normalizes the product of the power affected and the periods of supply disruption by the available power of the distribution network.

Fault detection in power distribution networks is a sequential process, i.e., the power line affected by the fault is sectorized, and each section is sequentially energized to locate the fault, which will be isolated to be finally repaired. Many different fault detection methods have been proposed, e.g., based on: signal processing (Othman and Amari, 2008), electrical methods (Saha et al., 2010), distributed control systems (Shames et al., 2011; Molzahn et al., 2017), artificial neural networks (Baqui et al., 2011; Jamil et al., 2015), and hypotheses testing (Dobakhshari and Ranjbar, 2015). Also, a good survey of feature extraction methods can be found in (Chen et al., 2016). Regardless of the method implemented, different switchgears take part to control, protect, and isolate the electrical equipment during the fault location/repair in a power line. 
In this work, we will focus on Transformation Centers (TCs), which can be classified as either Local Transformation Centers (LTCs) (with human maintenance teams) or Remote Control Transformation Centers (RCTCs) (remote management and supervision capabilities). The fault finding method with human maintenance teams is time inefficient, and causes an ITEIC increase. Nevertheless, the installation and mantainance of a high number of RCTCs is not cost efficient. This way, it is very important from an economic viewpoint to achieve a tradeoff between LTCs and RCTCs. Note that electricity corporations use in practice a sequential process to locate a fault.

The main contribution of this work is to introduce a planning method to find the best topology of RCTCs in distribution power lines. To this end, a refinement of the ITEIC supply quality indicator, with the aim of considering not only quality of service but also installation and operating costs, is proposed to define the characteristic fuction of a cooperative game. The Shapley value of this game (Shapley, 1953), which is the most well-known one point solution concept in cooperative game theory, will be used to measure the efficiency of RCTCs to be installed. The Shapley value has been applied in many different fields such as: biology (Lucchetti et al., 2010), finance applications (Tarashev et al., 2016), and system partitioning (Muros et al., 2018). Focusing on ranking purposes, the Shapley value has also been used in (Papapetrou et al., 2011), to rank individuals who collaborate in teams; (Kondratev and Mazalov, 2017), to elect candidates for a certain position based on ballots filled by voters; (Narayanam and Narahari, 2011), to discover a small set of influential players in a given social network; and (Zhang et al., 2014), to allocate carbon emission quotas among regions.

The remaining of the paper is organized as follows. In Section 2, the problem formulation is introduced. In Section 3, a novel cost index based on the ITEIC index to measure the best topology of RCTCs from both the viewpoint of costumer satisfaction and the company budget is proposed. The game theory perspective is presented in Section 4, where the Shapley value is considered to rank the RCTCs in terms of the newly proposed index. In Section 5, an example based on real data taken from a Spanish electricity company is used to test the method. Finally, conclusions and future lines of work are provided in Section 6.

\section{PROBLEM STATEMENT}

Consider a distribution power line, which is composed of a set of switchgear, e.g., reclosers, disconnectors, break switches, transformation centers (TCs), knife switches, etc. These elements can be manipulated either remotely or manually to locate, isolate, and repair a power fault. The goal here is to choose the best elements in this set to be remotely controlled with the aim of improving the quality of service at a reasonable cost. For simplicity, energy costs, maintenance costs, and the consumer prices index (CPI), are assumed to remain constant in this work.

For the sake of simplicity, we will assume that only a subset of the swithgear can be manipulated in the power fault location. We will work with a set of TCs, denoted by $\mathcal{N}=\{1,2, \ldots, N\}$, which will be located in strategic

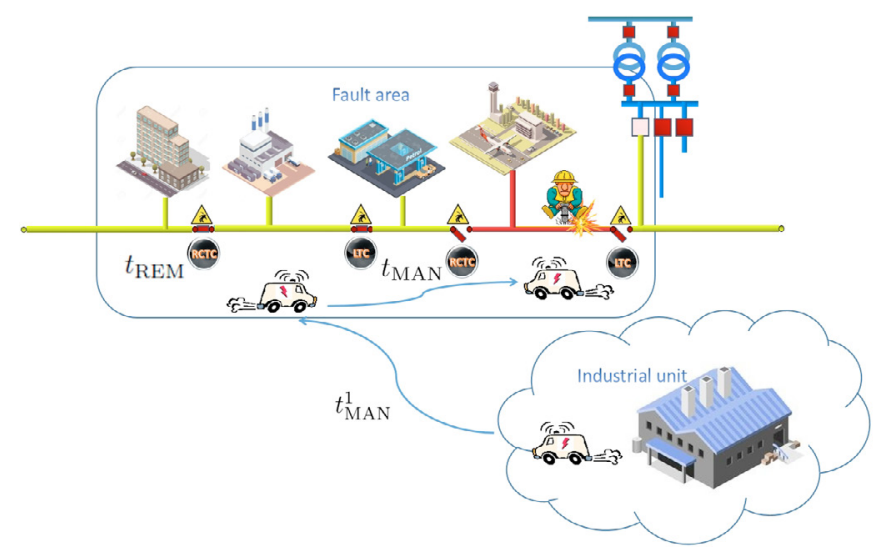

Fig. 2. Actuation time components

positions in the power line. The power capacity in the rest of the line is assumed to be uniformly distributed in every section. Notice that the elements inside set $\mathcal{N}$ can be either LTCs or RCTCs, being the subset of RCTCs symbolized by $\mathcal{S}$, with $\mathcal{S} \subseteq \mathcal{N}$. In this context, it can be seen that there are $2^{N}$ different configurations or topologies for set $\mathcal{S}$, depending on the elements of $\mathcal{N}$ included, and denoted as $\mathcal{S}_{0}, \mathcal{S}_{1}, \ldots, \mathcal{S}_{2^{N}-1}$. For instance, with $N=2$, there are four different topologies, i.e., $\mathcal{S}_{0}=\emptyset, \mathcal{S}_{1}=\{1\}, \mathcal{S}_{2}=\{2\}$ and $\mathcal{S}_{3}=\mathcal{N}=\{1,2\}$.

Likewise, in this work it is also assumed that the distribution lines are not able to be energized in case of energy lack or fault by any other power line. Also, we consider here that if there is at least one RCTC, it will be the first position to be maneuvered. Finally, notice that the actuation time, that is, the time spent to locate the fault, will depend on topology $\mathcal{S}$, which is intimately related to the presence of RCTCs and LTCs and therefore, the time it takes to maneuver on those devices. Hence, the actuation time will be influenced by the time spent in remote (manual) maneuvers - denoted by $t_{\mathrm{REM}}\left(t_{\mathrm{MAN}}\right)-$ and also by the time used by the maintenance team to move from the industrial unit to the fault area, which is included in the first manual maneuver $-t_{\mathrm{MAN}}^{1}-$, as shown in Fig. 2.

\subsection{Supply quality indicators in distribution networks}

Efficiency in power networks is directly related to the concept of quality of supply, which implies to distribute the electricity in the best conditions of voltage and frequency, while minimizing the number and duration of electric interruptions, which are directly perceived by customers. Focusing on the Spanish case, there are more restrictions to dealing with the duration rather than with the number of interruptions. In particular, the power interruption time is measured in Spain for distribution networks by means of the supply quality indicator named ITEIC ${ }^{1}$, defined legally as (Spanish Ministry of Economy, 2000)

$$
\text { ITEIC }=\frac{\sum_{j=1}^{f}\left(P^{j} \cdot H^{j}\right)}{P},
$$

1 This index is called TIEPI (Tiempo de Interrupción Equivalente a la Potencia Instalada) in Spanish. 
where $P$ is the total power capacity in a distribution power line in $\mathrm{kVA}, P^{j}$ is the power capacity affected by power interruption $j$ in $\mathrm{kVA}, H^{j}$ is the duration of power interruption $j$ in hours, and $f$ is the number of interruptions in the distribution power line.

\section{EFFICIENCY AND ECONOMY INDEX}

The ITEIC index is a legal index that only assesses the quality of supply from the viewpoint of customers and the government agencies. Nevertheless, it is not as useful for the company because it is only based on the total power capacity, i.e., no other information regarding the power line is considered. While the goal of any electricity corporation is to provide quality of service to customers, it must also be economically viable and sustainable along time. This way, both aspects should be jointly considered. To this end, a new cost index that measures the weighted time of power outages, the installation and maintenance costs, and also the physical features of the power line under study is provided in this section. This index specifically depends on topology $\mathcal{S} \subseteq \mathcal{N}$ (see Section 2), i.e., the number and location of RCTCs in the power line, and is defined as

$$
J(\mathcal{S})=J_{\mathrm{ins}}(\mathcal{S})+J_{\operatorname{man}}(\mathcal{S})+J_{\mathrm{loc}}(\mathcal{S})+J_{\text {rep }}(\mathcal{S}),
$$

where $J_{\text {ins }}(\mathcal{S}), J_{\text {man }}(\mathcal{S}), J_{\text {loc }}(\mathcal{S})$ and $J_{\text {rep }}(\mathcal{S})$ correspond respectively to the installation, maintenance, location and fault repair costs. Each term in $J(\mathcal{S})$ is calculated as follows:

$$
\begin{gathered}
J_{\text {ins }}(\mathcal{S})=c_{\mathrm{ins}}|\mathcal{S}|, \\
J_{\mathrm{man}}(\mathcal{S})=c_{\mathrm{man}} \cdot(y-1) \cdot|\mathcal{S}|, \\
J_{\mathrm{loc}}(\mathcal{S})=E_{\mathrm{loc}}(\mathcal{S}) \cdot y \cdot t_{\mathrm{f}} \cdot c_{\mathrm{kv}}, \\
J_{\mathrm{rep}}(\mathcal{S})=E_{\mathrm{rep}}(\mathcal{S}) \cdot y \cdot t_{\mathrm{f}} \cdot c_{\mathrm{kv}},
\end{gathered}
$$

with $c_{\text {ins }}$ and $c_{\text {man }}$ being respectively RCTCs instalation and maintenaince average costs, $c_{\mathrm{kv}}$ is the energy cost per $\mathrm{kVA} \cdot \mathrm{h}, y$ refers to the number of years assessed, $t_{\mathrm{f}}$ is the annual average rate of faults, and $E_{\text {loc }}(\mathcal{S}), E_{\text {rep }}(\mathcal{S})$ correspond respectively to the amount of energy not supplied during the electrical fault location and repair in $\mathrm{kVA} \cdot \mathrm{h}$, being defined as

$$
\begin{gathered}
E_{\mathrm{loc}}(\mathcal{S})=\sum_{i=1}^{n-1} P_{i} \cdot t_{i}(\mathcal{S}), \\
E_{\mathrm{rep}}(\mathcal{S})=P_{n} \cdot t_{\mathrm{r}},
\end{gathered}
$$

where $n$ is the number of stages, that is, the periods of time when there is no change in the number of affected costumers by the power outage. Likewise, $P_{i}$ refers to the power in $\mathrm{kVA}$ not supplied during stage $i, t_{i}(\mathcal{S})$ is the duration of stage $i$, and $t_{\mathrm{r}}$ is the average time to repair the fault during last stage $n$.

Note that index $J(\mathcal{S})$ is preferable for electricity companies because it not only considers the total power capacity and the interruptions duration as ITEIC does, but also power line features, and installation and operating costs.
Remark 1. Time $t_{i}(\mathcal{S})$ varies according to each stage of the location/repair process. In particular, it depends on whether the maneuver is either manually or remotely performed, and also on the time to reach the fault area, as commented in Section 2.

\section{GAME THEORY PERSPECTIVE}

Index $J(\mathcal{S})$ assesses a specific topology $\mathcal{S}$ in a given power line by means of its quality of service and economic efficiency. In this section, we use game theory tools in the line of previous works (Maestre et al., 2014; Muros et al., 2017) to find out which TCs are more relevant/dispensable regarding $J(\mathcal{S})$. In this context, let us consider pair $(\mathcal{N}, \boldsymbol{J})$ to define a cooperative game with transferable utility, with $\boldsymbol{J}=\left[J\left(\mathcal{S}_{0}\right), J\left(\mathcal{S}_{1}\right), \ldots, J\left(\mathcal{S}_{2^{N}-1}\right)\right]^{\mathrm{T}}$, and where $\mathcal{N}$ is the set of TCs inside the power line under study, and $J(\mathcal{S})$ corresponds to the characteristic function of the game, which assigns a value to each topology $\mathcal{S} \subseteq \mathcal{N}$.

Once the game is defined, it is possible to use a payoff rule to find a vector that specifies the benefit or cost that each player - TC - can reasonably expect from the game. In this context, it corresponds to the average value that the improvement of a certain location provides. In general, the best locations to become RCTCs will be associated with lower values in the payoff rule. In this work, we will use the Shapley value (Shapley, 1953), which assigns to $(\mathcal{N}, \boldsymbol{J})$ payoff vector $\phi(\mathcal{N}, \boldsymbol{J})$, defined for all $i \in \mathcal{N}$ as

$\phi_{i}(\mathcal{N}, \boldsymbol{J})=\sum_{\mathcal{S} \subseteq \mathcal{N}, i \notin \mathcal{S}} \frac{|\mathcal{S}| !(|\mathcal{N}|-|\mathcal{S}|-1) !}{|\mathcal{N}| !}[J(\mathcal{S} \cup\{i\})-J(\mathcal{S})]$,

that is, the marginal contribution of each player in $\mathcal{N}$ is averaged for all the possible permutations it can be part of. The Shapley value is characterized by the properties of Linearity, Efficiency, Dummy player, and Symmetric players (Shapley, 1953).

\subsection{Probability distributions}

As commented before, the Shapley value of each TC assumes that all parameters involved in $J(\mathcal{S})$ are fixed, so that the characteristic function only depends on the topology. These results can be generalized if parameters can vary. For example, let us consider that the average rate of faults $t_{\mathrm{f}}$ can change in a pre-specified interval, i.e.,

$$
t_{\mathrm{f}} \in\left[t_{\mathrm{f}}^{\min }, t_{\mathrm{f}}^{\max }\right] .
$$

This way, we compute index $J\left(\mathcal{S}, t_{\mathrm{f}}\right)$ by using $(2)$, and then the corresponding Shapley value for a large number $L$ of $t_{\mathrm{f}}$ evaluations, so that more general results are obtained. By this randomized approach, which in particular belongs to the family of Monte Carlo methods (Zio, 2013), probability distributions for the Shapley value evaluated by (9) are obtained, being the respective mean values

$$
\mu_{\phi_{i}}=\frac{1}{L} \sum_{s=1}^{L} \phi_{i}^{s}(\mathcal{N}, \boldsymbol{J}),
$$

which represent an interesting indicator of each TC's efficiency in a more general context. 


\section{AN ILLUSTRATIVE EXAMPLE}

In this section, we will test the method introduced in Section 3 by means of an overhead distribution power line, for it is the most unfavorable case from the viewpoint of fault location and repair duration.

We will consider that the line has three strategic TCs, namely TC1, TC2, and TC3, susceptible to be remotely controlled, resulting in eight possible topologies $\mathcal{S}$ for RCTCs, which are represented in Fig. 4. Note that these three locations divide the line into four different sections where the fault can be located, namely A1, A2, A3 and A4. Note that the size of the problem is small to be illustrative, allowing us to provide the computation of all topologies and the corresponding results in the limited space of the paper.

For the computation of index $J(\mathcal{S})$ for each topology $\mathcal{S}$, we use a period of study of $y=10$ years and the following real average data obtained from a Spanish electricity company: $c_{\text {ins }}=3500 €, c_{\operatorname{man}}=200 €, c_{\mathrm{kv}}=0.7 € / \mathrm{kVA} \cdot \mathrm{h}$ and $t_{i}(\mathcal{S})$, which has been calculated using verified data from maneuvers with different duration depending on whether it is a remote maneuver $\left(t_{\mathrm{REM}}=3 \mathrm{~min}\right)$ or a manual one $\left(t_{\text {MAN }}=26 \mathrm{~min}\right)$. Regarding the latter, we also account for the time needed by the maintenance team to move from the industrial unit to the fault area, i.e., $t_{\mathrm{MAN}}^{1}=62 \mathrm{~min}$, as commented in Section 2. Likewise, we assume that $t_{\mathrm{f}}$, i.e., the annual average rate of faults in each section, is normally distributed with mean 1.85 and standard desviation 0.40, and we will implement the Monte Carlo method explained in Section 4.1, considering $L=10000$ samples. These simulations have been performed using Crystal Ball by Oracle ${ }^{\circledR}$ (EPM Information Development Team, 2017), in a $3.20 \mathrm{GHz}$ Intel ${ }^{\circledR}$ Core i3-6100T, 8 GB RAM computer. The resulting Shapley value probability distributions are depicted in Fig. 3 .

Taking these data into account, the energy not supplied $E_{\text {loc }}(\mathcal{S})+E_{\text {rep }}(\mathcal{S})$ is depicted for each topology $\mathcal{S}$ and each section A1, A2, A3 and A4, in Figs. 5a, 5b, 5c and 5d, respectively. Notice that this energy corresponds to the area under the different curves and it is equal to the power not supplied times the interruption time.

At this point, it is possible to use the information regarding the energy not supplied shown in Fig. 5, and the real data mentioned before to calculate $J_{\text {man }}(\mathcal{S}), J_{\text {ins }}(\mathcal{S}), J_{\text {loc }}(\mathcal{S})$ and $J_{\text {rep }}(\mathcal{S})$ for each topology $\mathcal{S}$, which are drawn in Fig. 6

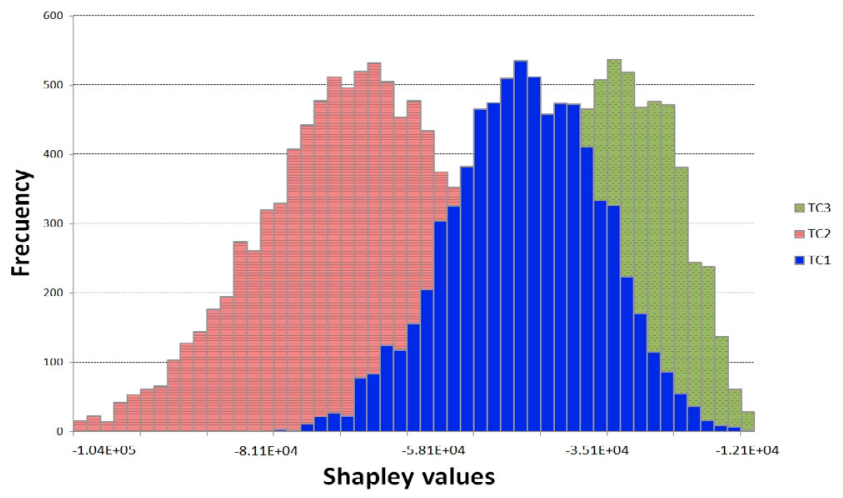

Fig. 3. Shapley value distributions for each TC
Table 1. Different costs (in $€$ ) for $t_{\mathrm{f}}=1.85$

\begin{tabular}{cc|c|c|c|c|c}
\multicolumn{2}{c|}{ Topology } & $J_{\text {loc }}(\mathcal{S})$ & $J_{\text {rep }}(\mathcal{S})$ & $J_{\text {ins }}(\mathcal{S})$ & $J_{\operatorname{man}}(\mathcal{S})$ & $J(\mathcal{S})$ \\
\hline $\mathcal{S}_{0}$ & $\emptyset$ & 5460 & 401200 & 0 & 0 & 406655.27 \\
$\mathcal{S}_{1}$ & $\{1\}$ & 5230 & 364050 & 3500 & 2000 & 374582.58 \\
$\mathcal{S}_{2}$ & $\{2\}$ & 5010 & 327110 & 3500 & 2000 & 337411.90 \\
$\mathcal{S}_{3}$ & $\{3\}$ & 4780 & 350360 & 3500 & 2000 & 360441.56 \\
$\mathcal{S}_{4}$ & $\{1,2\}$ & 4890 & 285400 & 7000 & 4000 & 300894.89 \\
$\mathcal{S}_{5}$ & $\{1,3\}$ & 5120 & 335680 & 7000 & 4000 & 351398.53 \\
$\mathcal{S}_{6}$ & $\{2,3\}$ & 4660 & 327850 & 7000 & 4000 & 343115.93 \\
$\mathcal{S}_{7}$ & $\mathcal{N}$ & 4100 & 252870 & 10500 & 6000 & 272862.49
\end{tabular}

and specifically detailed in Table 1 for the particular case of $t_{\mathrm{f}}=1.85$. Finally, with the aim of ranking the TCs for their remote management (to become RCTCs) in terms of economy and quality of supply, the mean values for the probability distributions in Fig. 3 have been taken, being the results for the case study of $y=10$ years detailed in bold letters in Table 2. Notice that the most suitable TC for remote management (RCTC) from the viewpoint of both the company and customers is TC2. Likewise, the second TC suggested is TC1, with TC3 being the last choice to be remotely controlled. These results stem from the average marginal contribution of each $\mathrm{TC}$ when it joins a random coalition.

Since this is a simple example with $N=3$ and eight topologies, it is possible to compare the eight values of $J(\mathcal{S})$ to find out which TC should became RCTC. Indeed, it can be seen in Table 1 that TC2 is the best choice if only one TC is going to be updated. Interestingly, TC3 seems to be a better option than TC1 when only one TC is upgraded, but

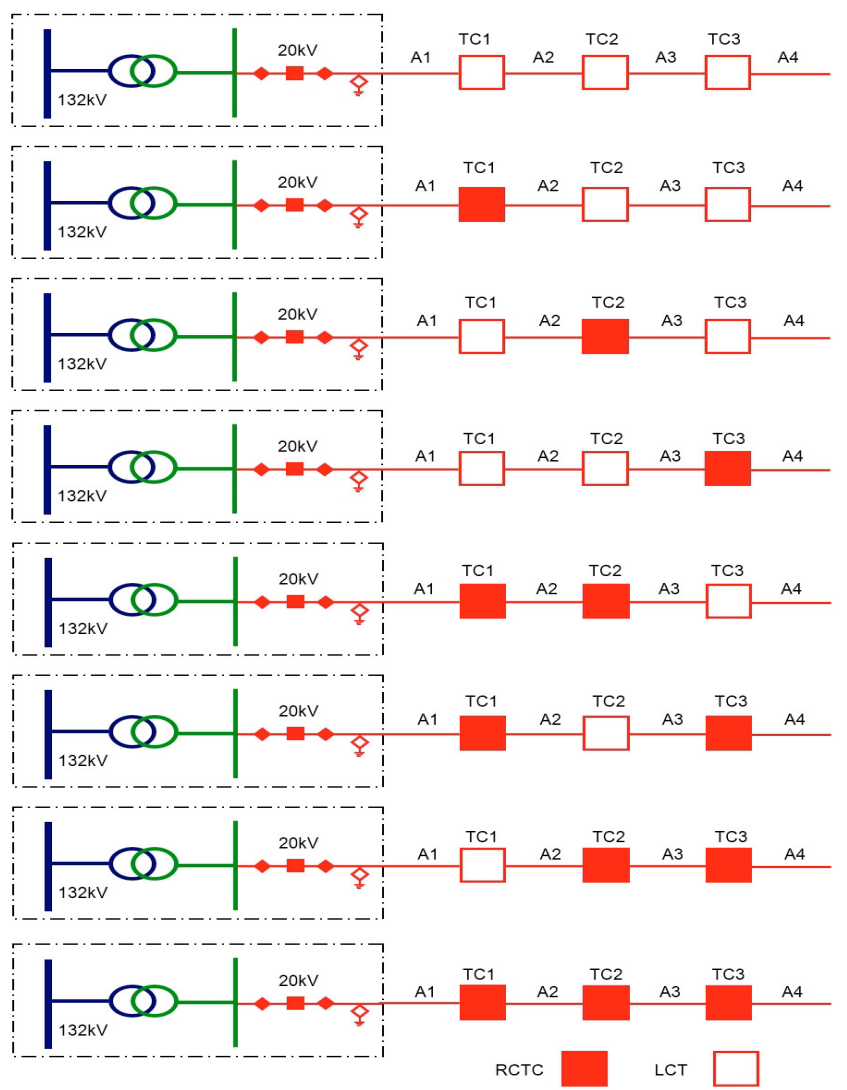

Fig. 4. Different topologies $\mathcal{S}$ depending on the number and position of LTCs and RCTCs 

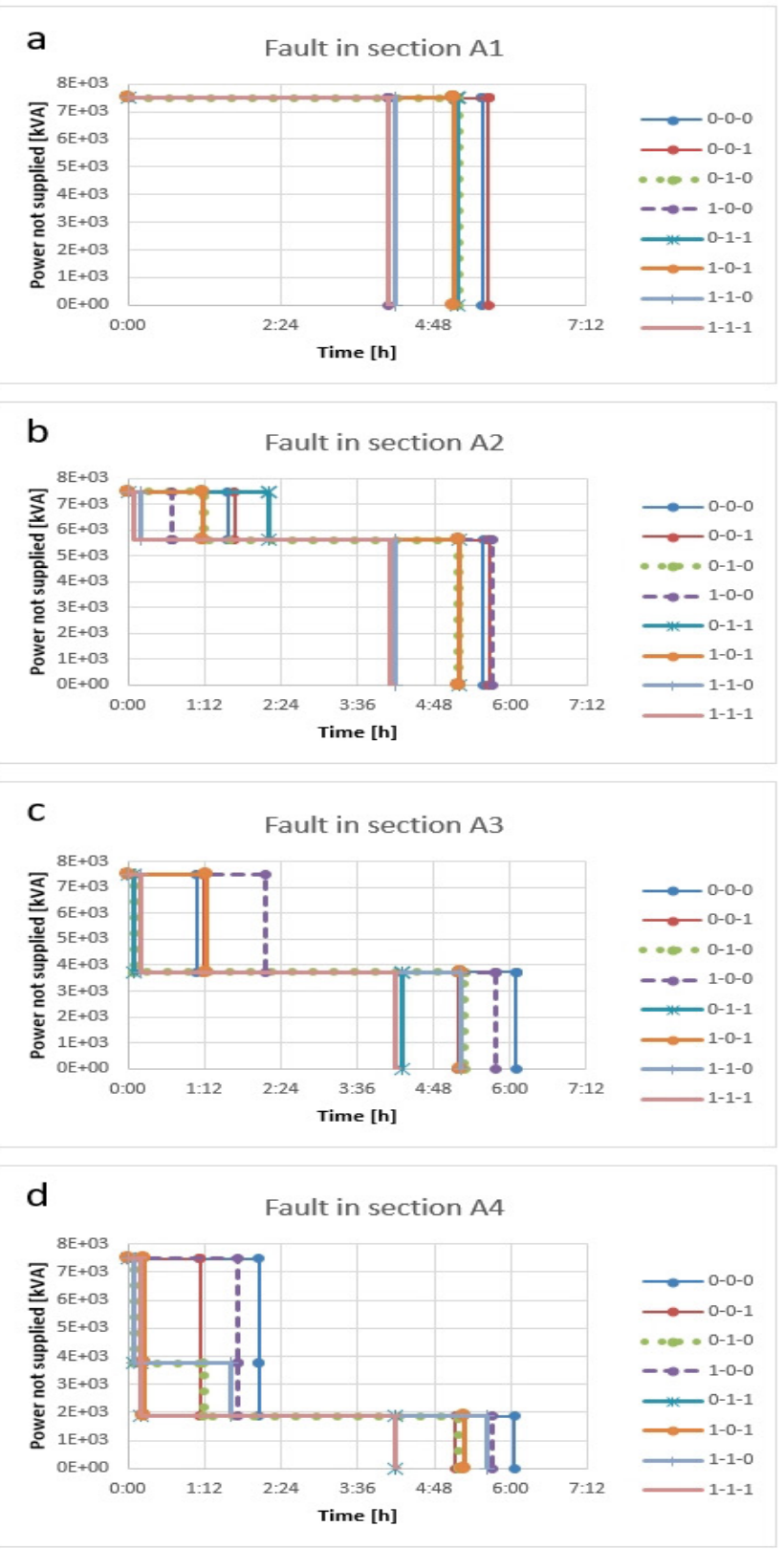

Fig. 5. Energy not supplied $E_{\text {loc }}(\mathcal{S})+E_{\text {rep }}(\mathcal{S})$ in $\mathrm{kVA} \cdot \mathrm{h}$, as a function of the topology and the section where the fault is located.

TC1 becomes a better complement for TC2 when two TCs are improved. As can be seen, the ranking of TCs based on the Shapley value captures this information. Also, note that when $N$ rises, the Shapley value becomes a valuable tool because it can be approximated by randomized methods (Castro et al., 2009), whereas it may not be possible to perform the explicit evaluation of all the topologies to find the optimal combination.

These results have been compared with those of considering different test durations, ranging from $y=1$ to 20 years, obtaining the evolution of the Shapley values detailed in Table 2 and depicted in Fig. 7. It can be seen that when the number of years rises, the difference between the Shapley values of the TCs increases significantly. Consequently, the benefit of remote-controlling any TC increases with time,
Table 2. Shapley value mean results for different periods of study

\begin{tabular}{|c|c|c|c|c|}
\hline & Years & TC1 & $\mathrm{TC} 2$ & TC3 \\
\hline \multirow{5}{*}{$\ddot{v}$} & 1 & $-1.1871 \times 10^{3}$ & $-3.4533 \times 10^{3}$ & $0.2130 \times 10^{3}$ \\
\hline & 2 & $-0.56790 \times 10^{4}$ & $-1.0214 \times 10^{4}$ & $-0.28774 \times 10^{4}$ \\
\hline & 5 & $-1.9236 \times 10^{4}$ & $-3.0617 \times 10^{4}$ & $-1.2206 \times 10^{4}$ \\
\hline & 10 & $-4.1750 \times 10^{4}$ & $-6.4501 \times 10^{4}$ & $-2.7696 \times 10^{4}$ \\
\hline & 20 & $-0.86442 \times 10^{5}$ & $-1.3177 \times 10^{5}$ & $-0.58440 \times 10^{5}$ \\
\hline
\end{tabular}

given that electricity corporations balance the losses due to maintenance and installation costs with the profits of the energy sold for the fast recovery of electricity supply, which in turn causes the economic revenue to improve, as can be seen in Fig. 7.

\section{CONCLUSIONS}

In this paper, a refinement of a legal Spanish supply quality indicator has been presented. This new index considers an economic tradeoff between final customers and electric companies interests. To this end, some strategic transformation centers (TCs) are assessed regarding the implementation of remote control capabilities.

The aforementioned problem has been analyzed from a game theory perspective, considering a game over the set of TCs, being its characteristic function associated with the proposed index. Then, the Shapley value of the game is taken into account to classify the TCs regarding their economic viability to become remote control TCs (RCTCs). The proposed index and the related Shapley value of the game have been computed in a simulation example where real data from a Spanish electricity company have been used. As our results show, the proposed method allows obtaining a relevance indicator to detect which TCs should become RCTCs, ranking them according to their impact regarding quality of supply, which is essential for customer satisfaction, and economic viability, which is also important for electricity corporations.

Future research should consider more complex and realistic distribution power networks with more TCs, by using randomized methods as that of Castro et al. (2009) to estimate the Shapley value. Likewise, the use of other lines to feed the line under study during power outages will also be taken into account. Finally, new methods to find the most interesting line to be improved in a given area, e.g., a substation, will be addressed.

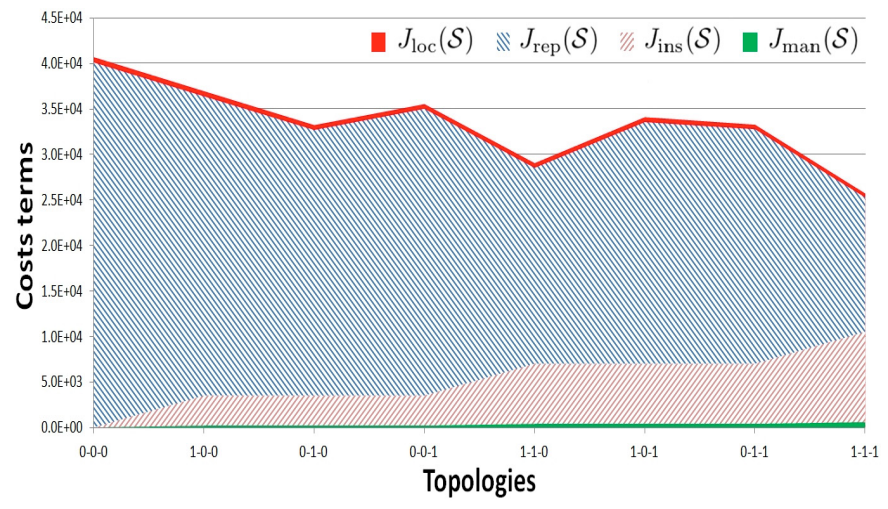

Fig. 6. Cost terms of index $J(\mathcal{S})$ for the different topologies involved 


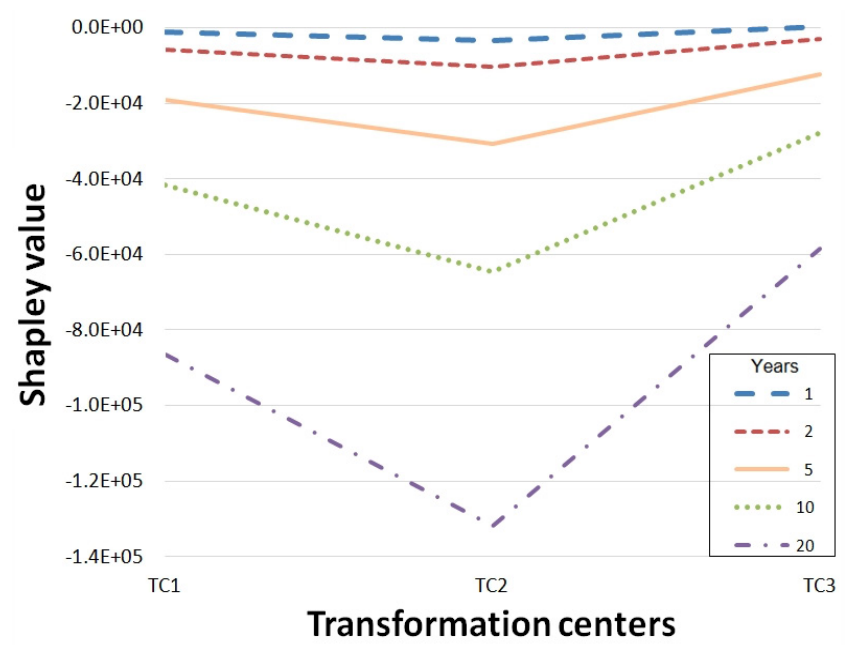

Fig. 7. Shapley values for different periods of study

\section{REFERENCES}

Baqui, I., Zamora, I., Mazón, J., and Buigues, G. (2011). High impedance fault detection methodology using wavelet transform and artificial neural networks. Electric Power Systems Research, 81(7), 1325-1333.

Brown, R.E. (2009). Electric Power Distribution Reliability. CRC Press, Boca Raton, Florida, USA, $2^{\text {nd }}$ edition.

Castro, J., Gómez, D., and Tejada, J. (2009). Polynomial calculation of the Shapley value based on sampling. Computers \&3 Operations Research, 36(5), 1726-1730.

Chen, K., Huang, C., and He, J. (2016). Fault detection, classification and location for transmission lines and distribution systems: a review on the methods. High Voltage, 1(1), 25-33.

Dobakhshari, A.S. and Ranjbar, A.M. (2015). A wide-area scheme for power system fault location incorporating bad data detection. IEEE Transactions on Power Delivery, 30(2), 800-808.

EPM Information Development Team (2017). Oracle $®$ Crystal Ball. Installation and Licensing Guide, v.11.1.2.4.850. https://www.oracle.com/middleware/ technologies/crystalball/downloads.html.

Gómez-Expósito, A., Conejo, A.J., and Cañizares, C. (2018). Electric Energy System-Analysis and Operation. Electric Power Engineering Series. CRC Press, Boca Raton, Florida, USA, $2^{\text {nd }}$ edition.

Grainger, J.J., Stevenson, W.D., and Chang, G.W. (2016). Power System Analysis. McGraw-Hill Education, New York, USA, $2^{\text {nd }}$ edition.

IEEE (2012). Guide for electric power distribution reliability indices. ieee std 1366-2012 (revision of ieee std. 1366-2003). DOI: 10.1109/IEEESTD.2012.6209381.

Jamil, M., Sharma, S.K., and Singh, R. (2015). Fault detection and classification in electrical power transmission system using artificial neural network. SpringerPlus, 4, 334.1-334.13.

Kondratev, A. and Mazalov, V. (2017). A ranking procedure with the Shapley value. In Proceedings of the $9^{\text {th }}$ Asian Conf. on Intelligent Information and Database Systems (ACIIDS 2017), 691-700. Kanazawa, Japan.

Lucchetti, R., Moretti, S., Patrone, F., and Radrizzani, P. (2010). The Shapley and Banzhaf values in microarray games. Computers \& Oper. Research, 37(8), 1406-1412.
Maestre, J.M., Muñoz de la Peña, D., Jiménez Losada, A., Algaba, E., and Camacho, E.F. (2014). A coalitional control scheme with applications to cooperative game theory. Optimal Control Applications and Methods, 35(5), 592-608.

Molzahn, D.K., Dörfler, F., Sandberg, H., Low, S.H., Chakrabarti, S., Baldick, R., and Lavaei, J. (2017). A survey of distributed optimization and control algorithms for electric power systems. IEEE Transactions on Smart Grid, 8(6), 2941-2962.

Muros, F.J., Maestre, J.M., Algaba, E., Alamo, T., and Camacho, E.F. (2017). Networked control design for coalitional schemes using game-theoretic methods. Automatica, 78, 320-332.

Muros, F.J., Maestre, J.M., Ocampo-Martinez, C., Algaba, E., and Camacho, E.F. (2018). A game theoretical randomized method for large-scale systems partitioning. IEEE Access, 6(1), 42245-42263.

Narayanam, R. and Narahari, Y. (2011). A Shapley valuebased approach to discover influential nodes in social networks. IEEE Transactions on Automation Science and Engineering, 8(1), 130-147.

Othman, M.F. and Amari, H.A. (2008). Online fault detection for power system using wavelet and PNN. In Proceedings of the IEEE $2^{\text {nd }}$ International Power and Energy Conference (PECON 2008), 1644-1648. Johor Bahru, Malaysia.

Papapetrou, P., Gionis, A., and Mannila, H. (2011). A Shapley value approach for influence attribution. In D. Gunopulos, T. Hofmann, D. Malerba, and M. Vazirgiannis (eds.), Machine Learning and Knowledge Discovery in Databases, volume 6912 of Lecture Notes in Computer Science, 549-564. Springer, Berlin, Germany.

Saha, M.M., Izykowski, J.J., and Rosolowski, E. (2010). Fault Location on Power Networks. Power Systems. Springer, London, UK.

Shames, I., Teixeira, A.M.H., Sandberg, H., and Johansson, K. (2011). Distributed fault detection for interconnected second-order systems. Automatica, 47(12), $2757-2764$.

Shapley, L.S. (1953). A value for $n$-person games. In H.W. Kuhn and A.W. Tucker (eds.), Contributions to the Theory of Games II. Annals of Mathematics Studies, vol. 28, 307-317. Princeton University Press, Princeton, New Jersey, USA.

Spanish Ministry of Economy (2000). Real decreto 1955/2000, de 1 de diciembre, por el que se regulan las actividades de transporte, distribución, comercialización, suministro y procedimientos de autorización de instalaciones de energía eléctrica. https://www.boe.es/eli/es/rd/2000/12/01/1955/con.

Tarashev, N., Tsatsaronis, K., and Borio, C. (2016). Risk attribution using the Shapley value: Methodology and policy applications. Review of Finance, 20(3), 11891213.

Zhang, Y.-J., Wang, A.-D., and Da, Y.-B. (2014). Regional allocation of carbon emission quotas in China: Evidence from the Shapley value method. Energy Policy, 74, 454464.

Zio, E. (2013). The Monte Carlo Simulation Method for System Reliability and Risk Analysis. Springer, London, UK. 